\title{
Acoustic Radiation Force Impulse Imaging (ARFI) on an IVUS Circular Array
}

\author{
Vivek Patel', Jeremy J. Dahl', David P. Bradway', \\ Joshua R. Doherty', Seung Yun Lee', and Stephen W. Smith'
}

\begin{abstract}
Our long-term goal is the detection and characterization of vulnerable plaque in the coronary arteries of the heart using intravascular ultrasound (IVUS) catheters. Vulnerable plaque, characterized by a thin fibrous cap and a soft, lipid-rich necrotic core is a precursor to heart attack and stroke. Early detection of such plaques may potentially alter the course of treatment of the patient to prevent ischemic events. We have previously described the characterization of carotid plaques using external linear arrays operating at $9 \mathrm{MHz}$. In addition, we previously modified circular array IVUS catheters by short-circuiting several neighboring elements to produce fixed beamwidths for intravascular hyperthermia applications. In this paper, we modified Volcano Visions 8.2 French, $9 \mathrm{MHz}$ catheters and Volcano Platinum 3.5 French, 20 $\mathrm{MHz}$ catheters by short-circuiting portions of the array for acoustic radiation force impulse imaging (ARFI) applications. The catheters had an effective transmit aperture size of $2 \mathrm{~mm}$ and $1.5 \mathrm{~mm}$, respectively. The catheters were connected to a Verasonics scanner and driven with pushing pulses of I $80 \mathrm{~V} p-p$ to acquire ARFI data from a soft gel phantom with a Young's modulus of $2.9 \mathrm{kPa}$. The dynamic response of the tissue-mimicking material demonstrates a typical ARFI motion of I to 2 microns as the gel phantom displaces away and recovers back to its normal position. The hardware modifications applied to our IVUS catheters mimic potential beamforming modifications that could be implemented on IVUS scanners. Our results demonstrate that the generation of radiation force from IVUS catheters and the development of intravascular ARFI may be feasible.
\end{abstract}

\section{Keywords}

cardiac, intravascular, ARFI, transducer, coronary

\section{Introduction}

Coronary atherosclerotic disease is the major cause of cardiovascular-related deaths in the United States. The disease results from initial vessel endothelial damage and progresses to the formation of fatty streaks and atherosclerotic plaques. Many cases of acute myocardial infarction and

'Department of Biomedical Engineering, Duke University, Durham, NC, USA

\section{Corresponding Author:}

Stephen W. Smith, Department of Biomedical Engineering, Duke University, I36 Hudson Hall, Durham, NC 27708, USA.

Email: ssmith@duke.edu 
sudden cardiac death directly result from the rupture of specific atherosclerotic plaques known as vulnerable plaques in the coronary arteries. As a result, clinical decision-making and the implementation of therapeutic strategies rely on an understanding of plaque morphology. ${ }^{1-4}$

Coronary plaque vulnerability, defined as susceptibility to disruption and leading to thrombosis, is directly linked to $60 \%$ to $70 \%$ of coronary events. These plaques have a characteristic soft tissue core surrounded by a tough, fibrous cap with a thickness ranging from $65 \mu \mathrm{m}$ to $700 \mu \mathrm{m}^{5-7}$ and have a lipid core area (in a transverse section of the artery) larger than $1 \mathrm{~mm}^{2}$ or a ratio of the lipid core area to the total plaque area greater than $20 \% .^{7}$ Although imaging modalities including angiography, intravascular optical coherence tomography (OCT), contrast-based computed tomography (CT), and magnetic resonance imaging (MRI) can be used to detect the presence of plaques, a cheap, minimally invasive vulnerable plaque classification technique has remained an elusive goal. ${ }^{8,9}$ Early implementations of B-mode ultrasonography have resulted in poor reproducibility due to both the subjective nature of gray scale differentiation between hyperechoic and hypoechoic regions and the limited contrast difference between different regions of the plaque. ${ }^{10,11}$

Recent studies have illuminated the use of photoacoustic imaging combined with intravascular ultrasound (IVUS) to determine plaque morphology. Catheter-based intravascular ultrasound allows for a real-time cross-sectional view of the vessel wall endothelium and atherosclerotic disease with image resolutions up to $30 \mu \mathrm{m}$. However, reliable differentiation of vulnerable plaque is not possible with IVUS imaging alone. ${ }^{12-14}$ Intravascular photoacoustic (IVPA) imaging was developed to overcome the limitations of IVUS, providing a more reliable method for plaque characterization. In this approach, localized short laser pulses produced by optical fibers are used to generate unique ultrasound waves in tissue. The generation of these distinct acoustic signals results from differences in the optical absorption and thermal expansion properties of tissues. An IVUS catheter serves as an acoustic detector and displays differences in tissue morphology based on the varying intensities of the received signals. Preliminary experiments have demonstrated feasibility for a combined IVPA/IVUS approach but have shown limitations in catheter flexibility with the incorporation of side-firing optical fibers on IVUS transducers. ${ }^{15,16}$

Acoustic radiation force impulse (ARFI) ultrasound provides an alternative method for imaging the mechanical properties of tissue. This method utilizes commercially available ultrasound scanners to generate short duration acoustic radiation forces. The radiation force $F$ applied to the tissue is a function of the speed of sound in the medium $(c)$, the absorption coefficient of the tissue $(\alpha)$, and the temporal average intensity of the acoustic beam $(I)$ at a given point (Equation 1$).{ }^{17}$

$$
F=\frac{2 \alpha l}{c} \text {. }
$$

Localized displacements resulting from these forces have been measured using B-mode ultrasound correlation techniques immediately after the production of the radiation force. The differences in displacement magnitudes demonstrate variations in tissue stiffness and can be used to determine the elastic moduli of tissues. ${ }^{17}$

Intracardiac echocardiography (ICE) ARFI imaging is currently used to monitor radio frequency ablations (RFA) and the stiffness of the myocardium through a cardiac cycle. ICE imaging catheters consist of side-firing phased arrays that can generate sufficient radiation forces to track displacements in cardiac tissue. Although ICE has shown promising results for characterizing tissue stiffness with the implementation of ARFI pulse sequences, the larger 8 to $10 \mathrm{Fr}$ size of the ICE catheter transducers prohibits endovascular imaging of plaques in the smaller coronary arteries. ${ }^{18-20}$

In our previous studies, we successfully distinguished a soft lesion in a vessel phantom (Figure 1a) and detected a carotid artery plaque in vivo (Figure 1b) with the use of external vascular ARFI imaging via a linear array operating with a push frequency of $4 \mathrm{MHz}$ and track 


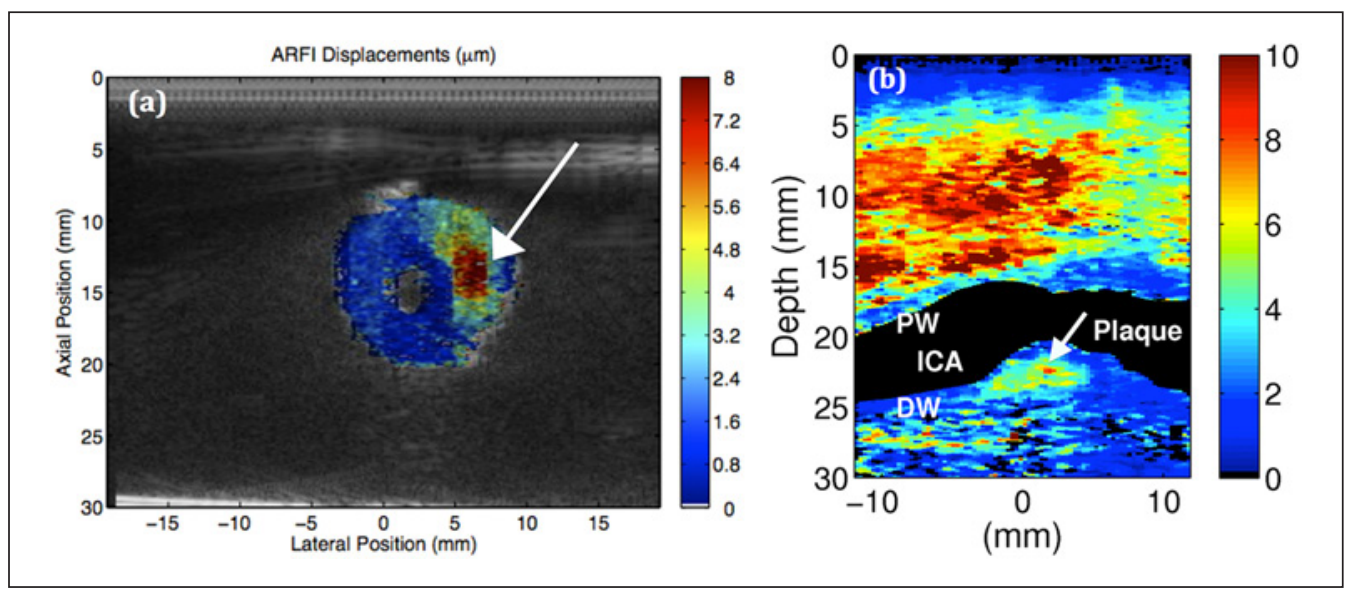

Figure I. (a) B-mode image overlaid with color ARFI results of an artery-mimicking tissue phantom with a soft lesion (white arrow). ${ }^{21}$ (b) Vascular ARFI image of a heterogeneous plaque with a soft region surrounded by a fibrous cap in a 55-year-old female volunteer. ${ }^{22}$

Note. ARFI = acoustic radiation force impulse imaging. Figure is available in full color in the online version at ultrasonicimaging. sagepub.com.

frequency of $6 \mathrm{MHz}$ on a Siemens S2000 ultrasound scanner. The in vivo result using the Siemens S2000 scanner shows a plaque with what may be a lipid core corresponding to regions of high displacements, a characteristic of soft tissues, and a tougher fibrous cap corresponding to regions of low displacements. ${ }^{21,22}$

The long-term purpose of this project is a minimally invasive, endovascular approach to image the mechanical properties of plaques in the coronary arteries. The design goal is a thin, flexible IVUS catheter that can provide real-time ARFI imaging in the lumen of small blood vessels that are not accessible to external vascular transducers. Circularly, phased array IVUS catheters operating at high frequencies of $20 \mathrm{MHz}$ contained within a sheath less than $4 \mathrm{Fr}$ have demonstrated efficacy in navigating coronary arteries with diameters of $2 \mathrm{~mm} .{ }^{23}$ As a result, the 3.5 Fr Eagle Eye Platinum Volcano transducer operating at $20 \mathrm{MHz}$ is considered here as a potential platform for IVUS ARFI imaging.

In this paper, we describe our first feasibility studies to evaluate the merits of intravascular ARFI imaging by modifying commercially available 3.5 Fr IVUS transducers to achieve appropriate power levels required for acoustic radiation force imaging. In order to validate our displacement results, we also used a $9 \mathrm{MHz}$ external linear array and the $8.2 \mathrm{Fr}, 9 \mathrm{MHz}$ Volcano Visions transducer as positive controls.

\section{Materials and Method}

\section{IVUS ARFI Prototypes}

For our prototypes, Volcano Visions and Volcano Eagle Eye Platinum imaging catheters (Volcano Corp., San Diego, California) were modified to accommodate higher voltages for ARFI as well as to connect to a Verasonics imaging system (Verasonics Inc., Redmond, Washington). Both transducers consist of a circular array of 64 piezoelectric elements attached to five ASIC chips operating at nominal frequencies of $9 \mathrm{MHz}$ and $20 \mathrm{MHz}$, respectively. Figure 2 shows short axis B-mode images of the same vessel phantom as in Figure 1a using the Volcano s5 ultrasound scanner. Note that the conventional B-scans show no hint of the soft lesion. 


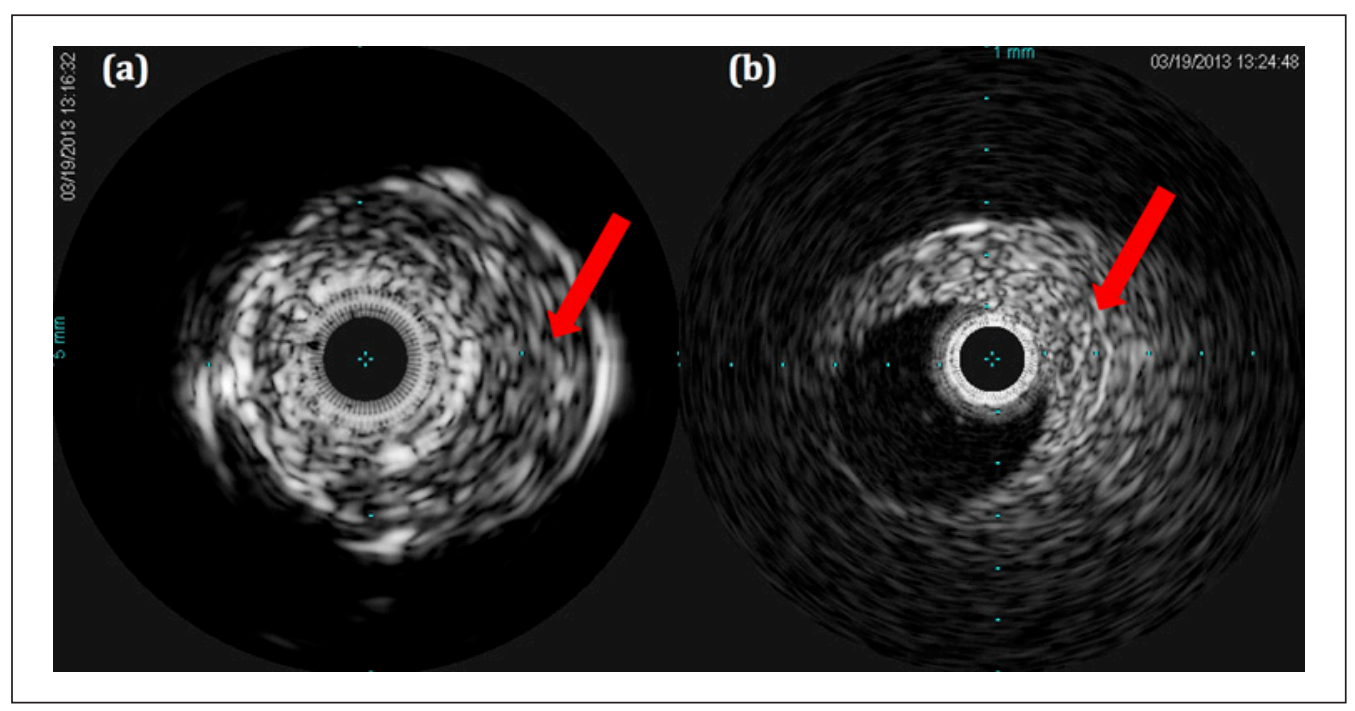

Figure 2. Vessel phantom images with the soft lesion indicated with the red arrows. (a) Volcano Visions $9 \mathrm{MHz}$ image. Image radius is $10 \mathrm{~mm}$. (b) Volcano Eagle Eye Platinum $20 \mathrm{MHz}$ image. Image radius is $6 \mathrm{~mm}$. Note. Figure is available in full color in the online version at ultrasonicimaging.sagepub.com.

Volcano IVUS circular array catheters are focused using conventional phased array techniques so that an $\sim 1 \mathrm{~mm}$ aperture can be focused at a depth of $1 \mathrm{~mm}$. Ideally, software modifications could be used to electronically phase a portion of the elements to both generate a focused acoustic radiation force beam and track the resulting displacements through time with the IVUS catheters. These modifications would allow for increased power levels and an efficient implementation of the ARFI imaging sequence for clinical applications; however, we did not have access to the Volcano scanner software and were limited to a maximum of $12 \mathrm{~V}$ transmit bursts due to the integrated circuits in the catheter transducers.

Therefore, in our experimental approach, we modified the transducers by physically shortcircuiting 16 elements, and we constructed a custom connection to a Verasonics imaging system. The Verasonics scanner supports higher power levels with a $192 \mathrm{~V}$ peak-to-peak (pk-pk) maximum, allows for the implementation of the ARFI imaging sequence, and provides access to the raw radio frequency $(\mathrm{RF})$ data for offline processing. Each transducer prototype functioned as a single element transducer to obtain one line of ARFI data. Our goal was to prove initial ARFI feasibility, even though the diverging acoustic wave produced by these modifications limit the available radiation force, which would greatly improve with electronic focusing of the elements. We have previously used this transducer approach to demonstrate ultrasound hyperthermia using IVUS arrays for intracranial applications. ${ }^{24}$ This paper builds on our previous work in developing 3-D ultrasound catheters. ${ }^{25-28}$

Much of the catheter was cut away leaving a total length of approximately $8 \mathrm{~cm}$. The traces to the circular array elements originated from five flex circuit boards in groups of 13. As shown in Figure 3, the polyimide surface covering the transducer elements was partially removed by making a cut close to the flex circuit board and carefully peeling back the polyimide layer to expose the traces. Silver paint was applied between the exposed traces to create a short circuit between 16 elements and a single silver wire was attached to the connected traces with silver epoxy (red arrow). A ground connection was established using the same technique at the opposite end of the elements (black arrow). The hot and ground silver wires were then attached to a micro-coaxial cable with a length of $40 \mathrm{~cm}$ and connected to the Verasonics V-1 scanner. 

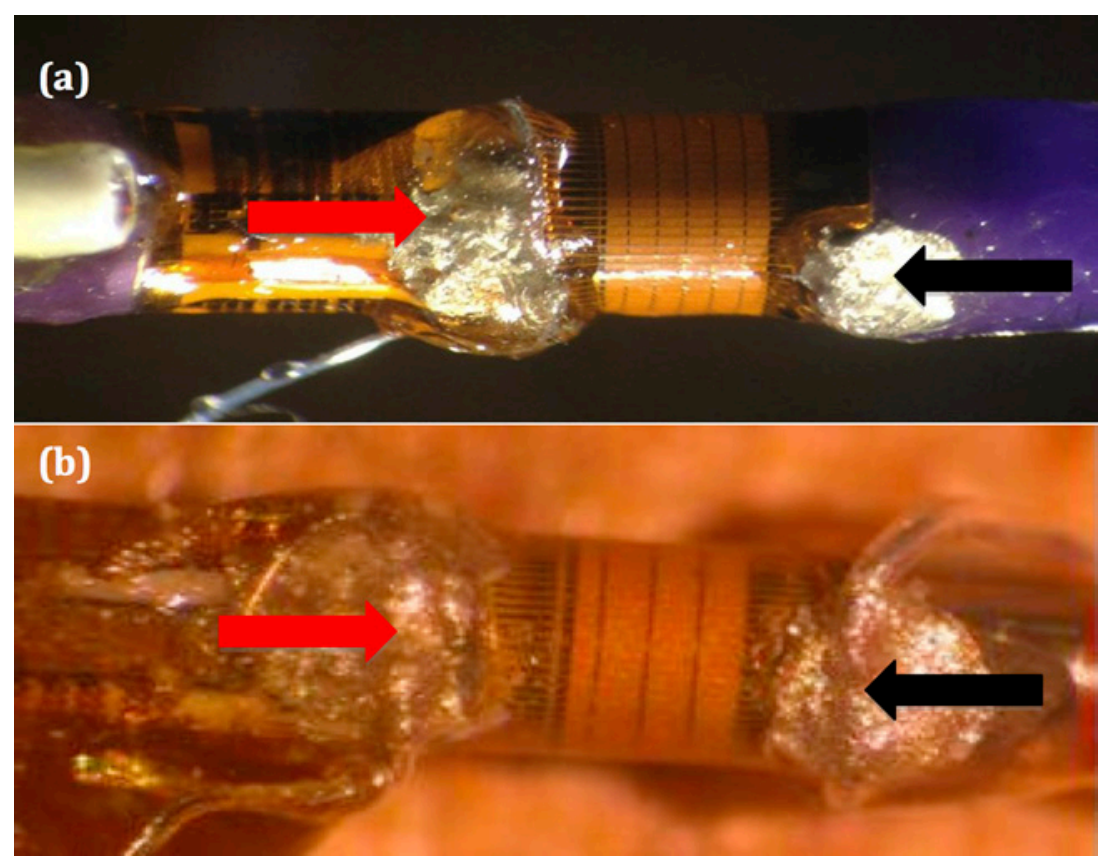

Figure 3. Images show IVUS prototypes with gold piezoelectric elements. Black lines extending from the elements represent the traces. Red arrow indicates hot connection and black arrow represents ground connection. (a) Volcano Visions prototype. (b) Volcano Eagle Eye Platinum prototype.

Note. IVUS = intravascular ultrasound. Figure is available in full color in the online version at ultrasonicimaging.sagepub. com.

Spectral analysis of each transducer prototype was then performed in a water tank with an impulse excitation using the Panametrics Pulser/Receiver Model 5073 PR (Olympus, Waltham, Massachusetts) to verify the specified nominal operating frequencies. Figure 4 illustrates power spectrum results for typical catheter transducer prototypes.

\section{Measurements}

The transmit beamplots of several such modified Volcano Eagle Eye Platinum catheters have been previously measured at a depth of $3 \mathrm{~mm}$ and described yielding a $-6 \mathrm{~dB}$ beamwidth of approximately 55 degrees. ${ }^{29}$ For our current measurements, the experimental setup is illustrated in Figure 5 wherein we sequentially measured ultrasound intensities and ARFI response in a tissue phantom as the Verasonics scanner drove the ATL linear array, Volcano Visions catheter, and Volcano Eagle Eye catheter.

For the intensity experiments, the transducer was held stationary $3 \mathrm{~mm}$ above a parallel hydrophone platform that could be adjusted on all three axes in a water tank. A calibrated membrane hydrophone (S4-251, Sonora Medical Systems, Longmont, Colorado) was used to capture a 10-cycle excitation beam from the Verasonics system. The linear array ATL L12-5 transducer with a $1.6 \mathrm{~mm}$ aperture operating at $9 \mathrm{MHz}$ was used as a positive control with a transmit voltage of $80 \mathrm{~V}_{\text {pk-pk}}$. The modified Volcano Visions catheter operating at $9 \mathrm{MHz}$ was then assessed with a transmit voltage of $192 \mathrm{~V}_{\mathrm{pk}-\mathrm{pk}}$, and finally, the modified Volcano Eagle Eye Platinum catheter operating at $18 \mathrm{MHz}$ was assessed with a transmit voltage of $192 \mathrm{~V}_{\mathrm{pk}-\mathrm{pk}}$. The $18 \mathrm{MHz}$ transmit 

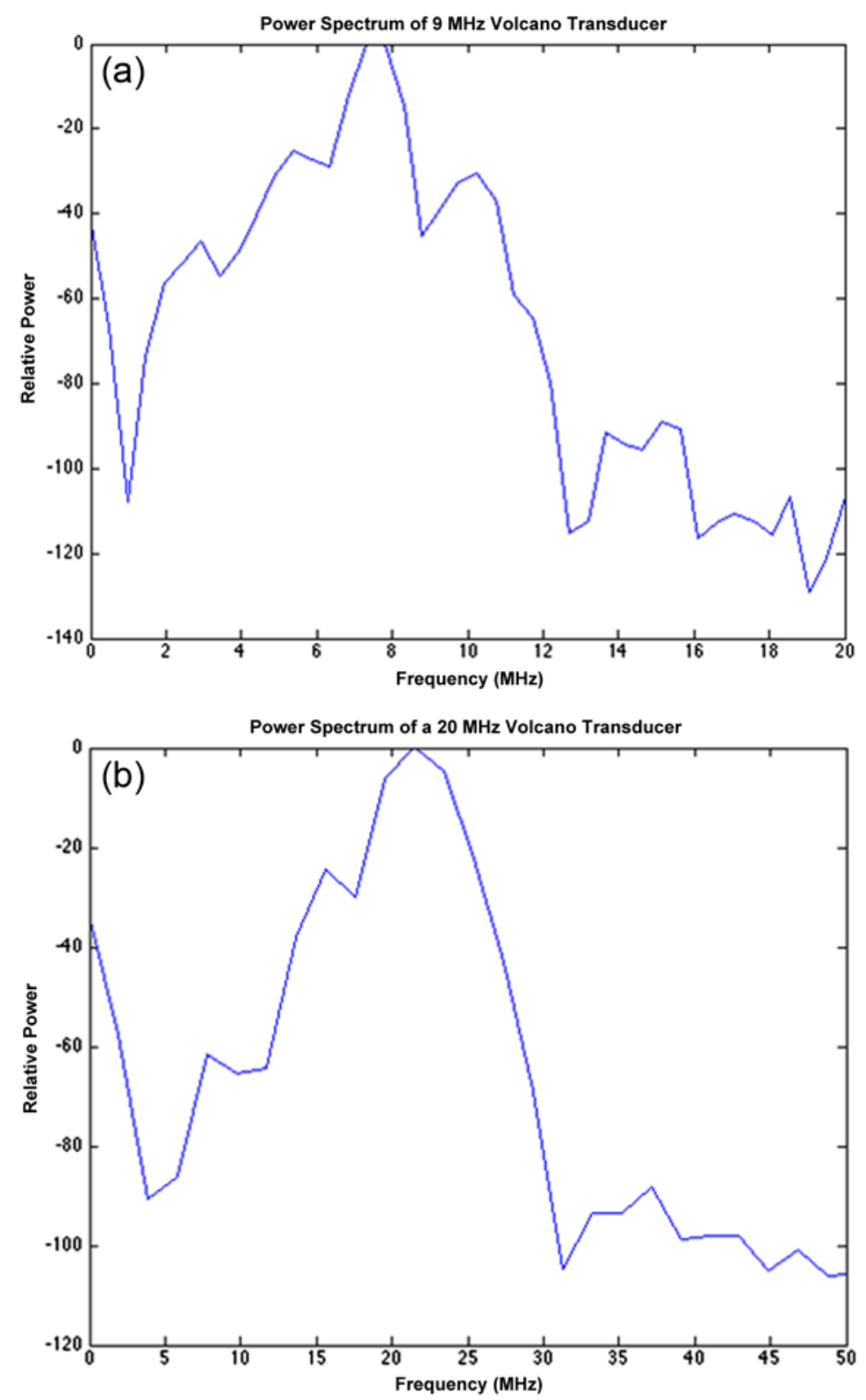

Figure 4. Pulse-echo power spectra were obtained from aluminum block reflection at a depth of 2.8 $\mathrm{mm}$ in a water tank. (a) Frequency spectrum for Volcano Visions prototype with a peak at $8 \mathrm{MHz}$. (b) Frequency spectrum for Volcano Eagle Eye Platinum prototype with a peak at $20.5 \mathrm{MHz}$. Note. Figure is available in full color in the online version at ultrasonicimaging.sagepub.com.

frequency of the Volcano Eagle Eye Platinum was used because of limitations on the available transmit frequencies for the Verasonics scanner. The voltage data were converted to pressures using the hydrophone calibration coefficients at the appropriate center frequencies. The pressure 


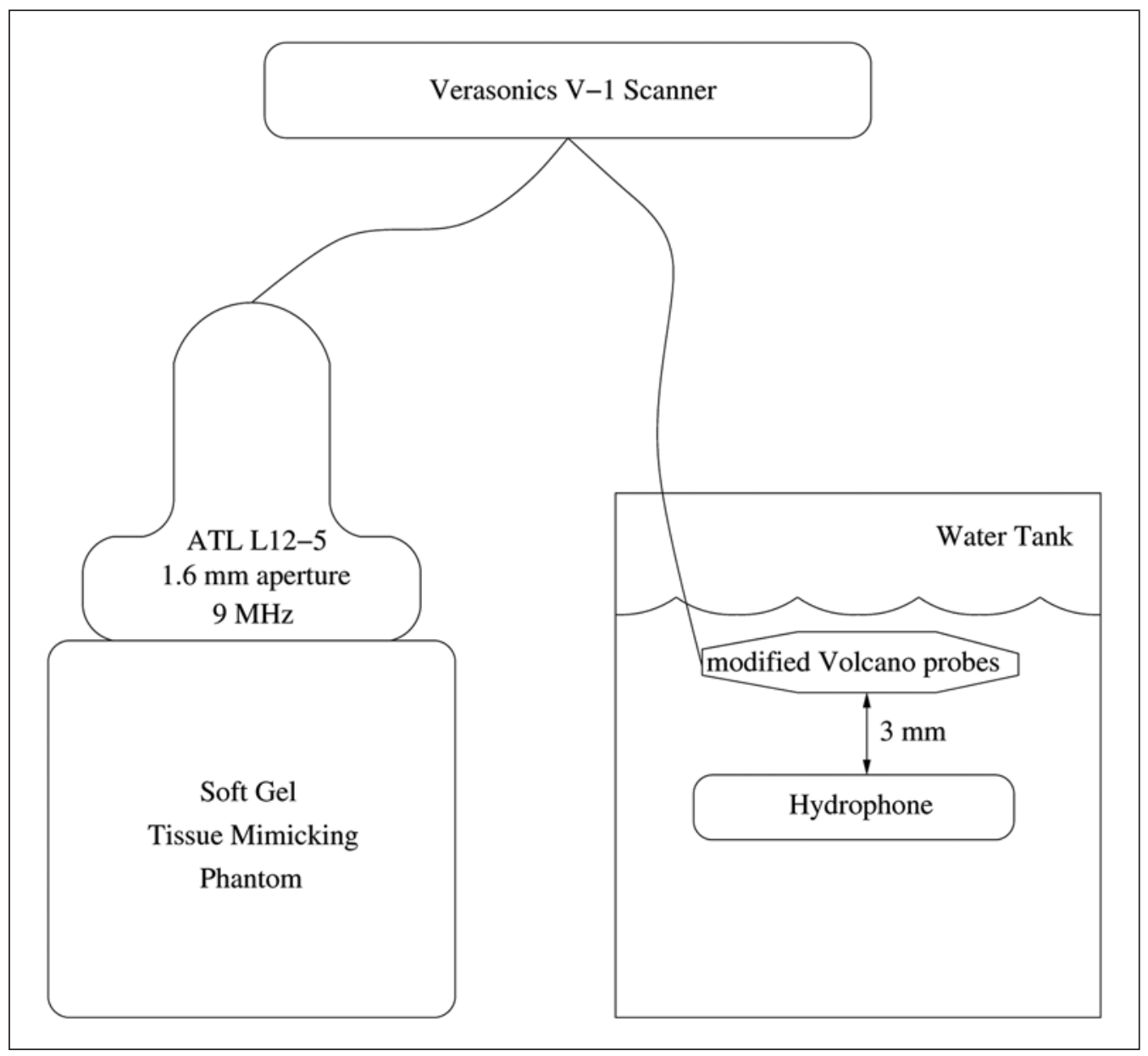

Figure 5. Block diagram of laboratory experiments showing the Verasonics scanner, the ATL LI2-5 transducer with a $1.6 \mathrm{~mm}$ aperture, and the modified Volcano catheters for hydrophone measurements and ARFI measurements in a tissue phantom.

Note. ARFI = acoustic radiation force impulse imaging.

amplitudes measured by the hydrophone for these waveforms were 5.77, 4.72, and 4.84 MPa for the ATL L12-5, Volcano Visions, and Volcano Eagle Eye Platinum catheter, respectively. The intensity spatial peak pulse average $\left(I_{\mathrm{SPPA}}\right)$ for each transducer was calculated using Equation 2 where $n$ is the number of cycles, $T$ is the period, $p$ is the pressure amplitude, $\rho$ is the density of the medium, and $c$ is the speed of sound through the medium. ${ }^{30}$ The nonderated $I_{\mathrm{SPPA}}$ values measured in water $(c=1490 \mathrm{~m} / \mathrm{s}, \rho=1 \mathrm{~g} / \mathrm{mL})$ for the ATL L12-5, modified Volcano Visions catheter, and modified Eagle Eye Platinum catheter were found to be $1120 \mathrm{~W} / \mathrm{cm}^{2}, 746 \mathrm{~W} / \mathrm{cm}^{2}$, and 786 $\mathrm{W} / \mathrm{cm}^{2}$, respectively. The nonderated mechanical indices (MI) for the transducers at these intensities was estimated to be 1.92 for the ATL L12-5, 1.57 for the modified Volcano Visions catheter, and 1.14 for the modified Eagle Eye Platinum catheter.

$$
I_{\mathrm{SPPA}}=\frac{1}{n T} \int_{0}^{n T} \frac{p^{2}}{\rho c} d t .
$$




\section{Phantom Fabrication}

The ARFI experiments were performed in elastography phantoms fabricated from gelatin, alcohol, gluteraldehyde, water, and graphite. Three phantoms were constructed using the recipe described in Nightingale et al. ${ }^{17}$ Each phantom was uniform cylinder approximately $8 \mathrm{~cm}$ in diameter and $8 \mathrm{~cm}$ deep, large enough to avoid boundary effects, with a Young's modulus around $3 \mathrm{kPa}$, which is in the range of moduli found in the soft tissue of atherosclerotic plaques. ${ }^{22} \mathrm{We}$ used shear wave imaging and the radon sum transformation method explained in Rouze et al. to determine the Young's modulus for each phantom. ${ }^{31,32}$

\section{Verasonics Data Acquisition}

For each ARFI experimental trial, the transducer was placed directly on the surface of a gelatin phantom immersed in mineral oil. The pulse sequence was designed to first acquire five reference A-lines followed by a pushing pulse at the same location. This pushing pulse refers to an extended duration pulse that provides the acoustic radiation force to displace the tissue. Immediately after the pushing pulse, a series of radio-frequency (RF) A-lines, referred to as tracking lines, were acquired in order to track the resulting displacements through time using a normalized cross-correlation algorithm. A pulse repetition frequency (PRF) of $5 \mathrm{kHz}$ was used for the pulse sequence and the raw RF data were stored for further processing. ${ }^{17,22}$

The ATL L12-5 linear array transducer operating at $9 \mathrm{MHz}$ was used as a positive control for these experiments. The pushing pulse had a duration of $107 \mu \mathrm{s}$ at a transmit voltage of 80 $\mathrm{V}_{\mathrm{pk}-\mathrm{pk}}$. Eighteen A-lines were acquired at a transmit voltage of $15 \mathrm{~V}_{\mathrm{pk}-\mathrm{pk}}$ over a duration of 3.5 ms. For a comparison to the smaller apertures of modified catheter transducer prototypes, the aperture of the ATL probe was incrementally reduced from 10 to $1.6 \mathrm{~mm}$ by varying the F-number (i.e., the number of active elements) and focal depth using software controls on the Verasonics scanner.

The modified Volcano Visions prototype was used to obtain single line ARFI data at 9 $\mathrm{MHz}$ and used a pushing pulse with a duration of $107 \mu \mathrm{s}$ at a transmit voltage of $192 \mathrm{~V}_{\mathrm{pk} \text {-pk }}$. The modified Volcano Eagle Eye Platinum prototype was used to obtain single line ARFI data at $18 \mathrm{MHz}$ with a pushing pulse duration of $72 \mu \mathrm{s}$ at a transmit voltage of $192 \mathrm{~V}_{\mathrm{pk} \text {-pk }}$. For both catheters, RF A-lines were acquired at a transmit voltage of $60 \mathrm{~V}_{\mathrm{pk}-\mathrm{pk}}$. Thirty tracking lines were acquired over $6 \mathrm{~ms}$, and the experiment was repeated for three different phantoms in each case.

\section{Data Processing}

Raw RF data were acquired from the Verasonics system at a sampling rate of $36 \mathrm{MHz}$ for the $9 \mathrm{MHz}$ transducers and a sampling rate of $72 \mathrm{MHz}$ for the Volcano Eagle Eye Platinum transducer prototype. For the ATL L12-5 transducer, the RF data were beamformed using a speed of sound of $1540 \mathrm{~m} / \mathrm{s}$. To track displacements in the typical range of 1 to 10 microns, the RF data were up-sampled by a factor of 5 to $180 \mathrm{MHz}$. Normalized cross correlation was implemented for each axial depth sequentially between the up-sampled reference and tracking lines. ${ }^{33}$ Kernel lengths corresponding to 1.5 wavelengths were used for the algorithm. The axial tissue displacements were estimated from the shift in the peak of the cross-correlation function between subsequent tracks, and the results were used to generate a model for the temporal response of the tissue-mimicking phantom after the application of the radiation force. A five-point moving median filter was implemented to smooth the data while preserving the maximum displacement amplitude. 


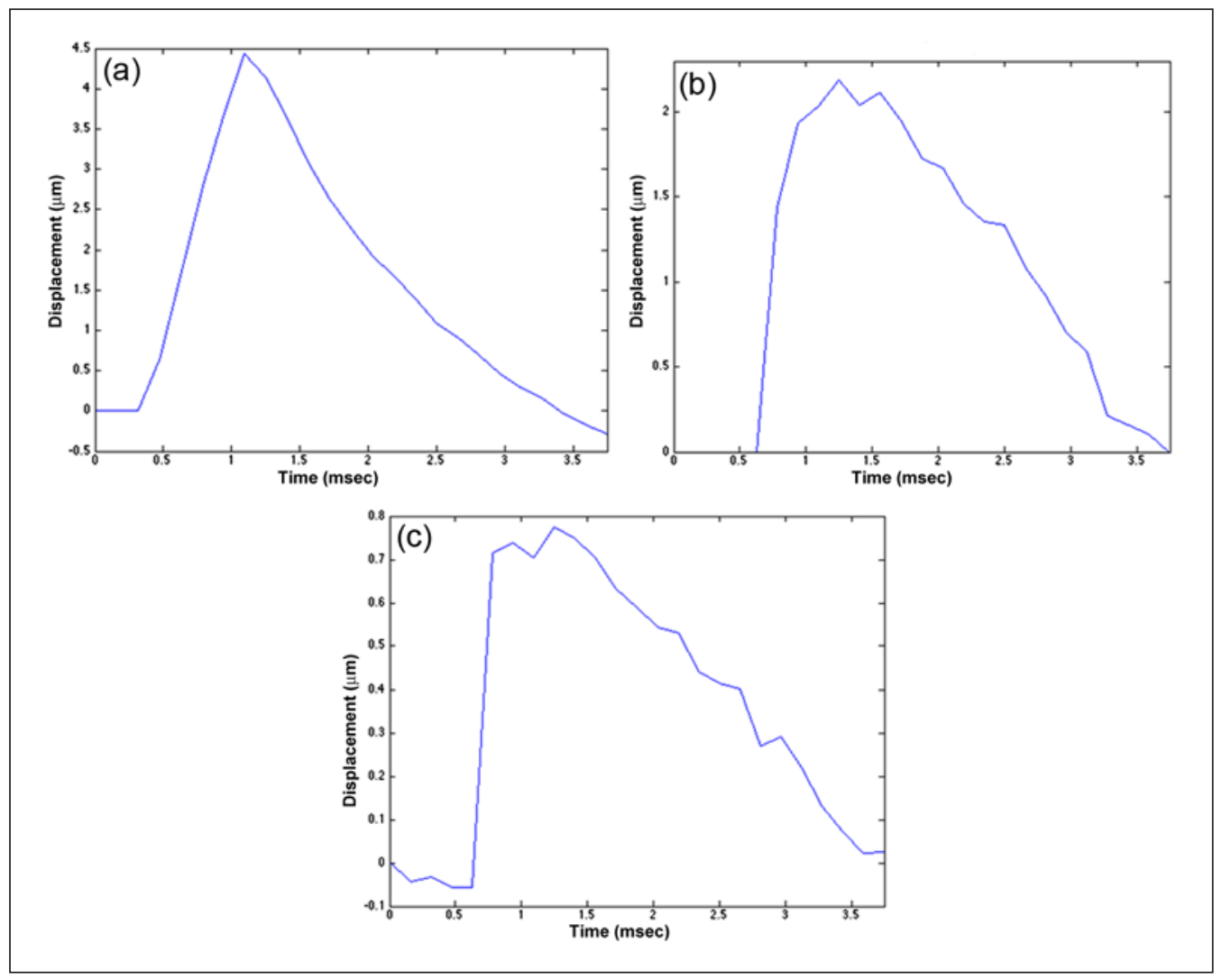

Figure 6. The graphs represent tissue displacement profiles through time at the axial focus for varying transmit apertures from the ATL LI2-5 linear array. (a) $10 \mathrm{~mm}$ aperture and $10 \mathrm{~mm}$ focus. (b) $5 \mathrm{~mm}$ aperture and $5 \mathrm{~mm}$ focus. (c) $1.6 \mathrm{~mm}$ aperture and $1.6 \mathrm{~mm}$ focus.

Note. Figure is available in full color in the online version at ultrasonicimaging.sagepub.com.

\section{Results}

\section{Linear Array ARFI Results}

The ATL L12-5 linear array was able to achieve sufficient ARFI displacement results in a $2.9 \mathrm{kPa}$ uniform gelatin phantom with apertures, varied under software control, ranging from 10 to 1.6 $\mathrm{mm}$. Figure 6 displays representative displacement profiles as a function of time for F/1 apertures. The three different apertures of $10 \mathrm{~mm}, 5 \mathrm{~mm}$, and $1.6 \mathrm{~mm}$ generated maximum displacement values of $4.5 \mu \mathrm{m}, 2.6 \mu \mathrm{m}$, and $0.78 \mu \mathrm{m}$, respectively.

\section{IVUS Prototype ARFI Results}

Both of the IVUS prototypes were able to successfully track ARFI displacements in three independent uniform gelatin phantoms (Phantoms A, B, and C). Figure 7 portrays illustrative displacement curves versus time for the $9 \mathrm{MHz}$ Volcano Visions prototype at an axial depth of 4 $\mathrm{mm}$. Ten trials at varying locations were conducted for each phantom, and the results are summarized in Table 1. The mean maximum displacement values for Phantoms A, B, and C were found to be $2.2 \mu \mathrm{m}, 2.08 \mu \mathrm{m}$, and $1.9 \mu \mathrm{m}$, respectively. 


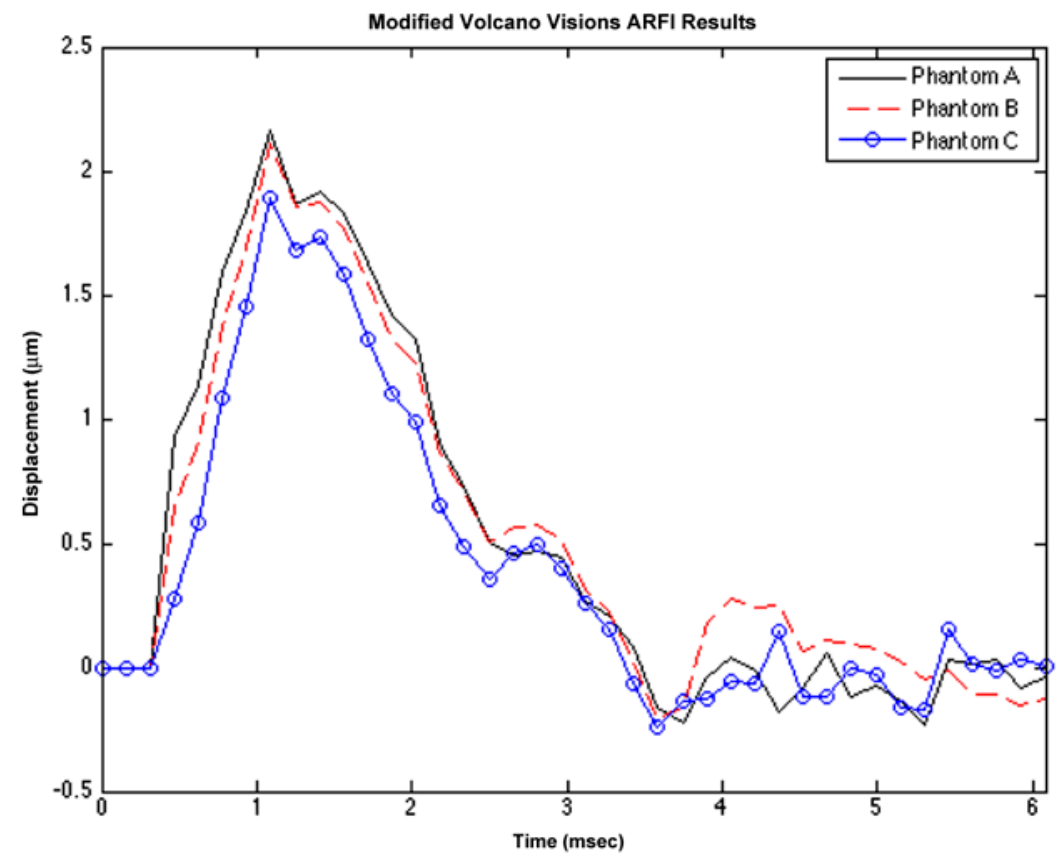

Figure 7. The graph depicts averaged ARFI displacement profiles for three phantoms with nearly identical elastic moduli. This illustrates the reproducibility of the displacement measurements for the Volcano Visions transducer prototype.

Note. ARFI = acoustic radiation force impulse imaging. Figure is available in full color in the online version at ultrasonicimaging. sagepub.com.

Table I. The Mean Maximum Displacement and Standard Deviation Values for $n=10$ Trials. Elastic Moduli of the Three Tissue-mimicking Phantoms are Reported as Well.

\begin{tabular}{lccc}
\hline & Phantom A $(2.6 \mathrm{kPa})$ & Phantom B $(2.8 \mathrm{kPa})$ & Phantom C $(2.9 \mathrm{kPa})$ \\
\hline $\begin{array}{l}\text { Mean maximum } \\
\text { displacement }(\mu \mathrm{m})\end{array}$ & 2.2 & 2.08 & 1.9 \\
$S D$ & 0.27 & 0.12 & 0.14 \\
\hline
\end{tabular}

Figure 8 shows an illustrative displacement profiles for the Volcano Eagle Eye Platinum prototype at an axial depth of $1.5 \mathrm{~mm}$. Ten trials were conducted for each phantom, and the results are summarized in Table 2. The mean maximum displacement values for Phantoms A, B, and C were found to be $0.65 \mu \mathrm{m}, 0.57 \mu \mathrm{m}$, and $0.55 \mu \mathrm{m}$, respectively. We note the skewed temporal response for Phantom A, which may be a result of poor SNR and areas of nonuniform speckle in the tissue-mimicking phantom.

\section{Discussion and Conclusion}

Volcano Visions and Volcano Eagle Eye Platinum IVUS catheters were modified to create prototypes for IVUS ARFI applications. Ideally, a portion of the elements would be electronically 


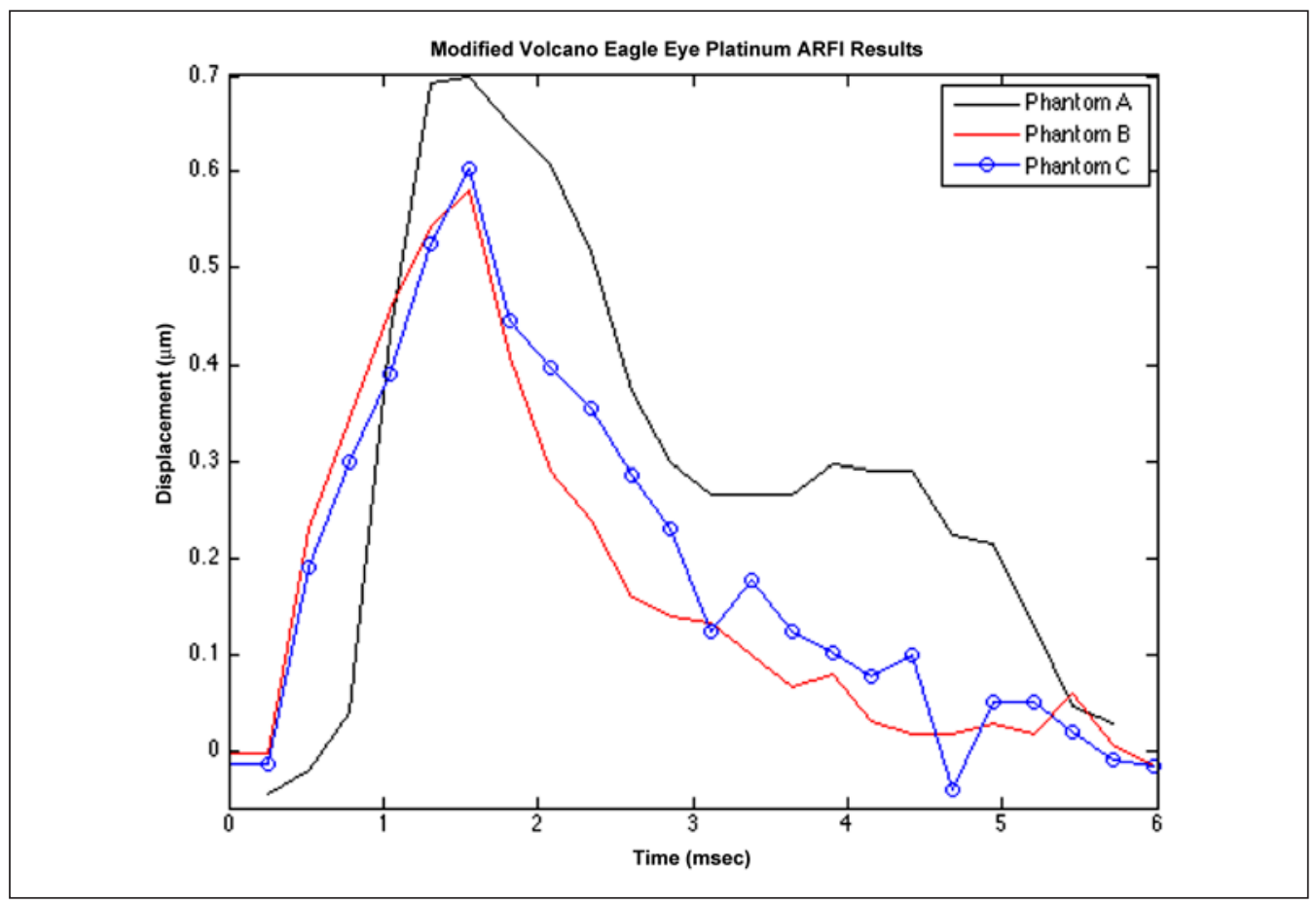

Figure 8. The graph depicts the representative ARFI displacement profiles for three phantoms with nearly identical elastic moduli. This illustrates the reproducibility of the displacement measurements for the Volcano Eagle Eye Platinum transducer prototype.

Note. ARFI = acoustic radiation force impulse imaging. Figure is available in full color in the online version at ultrasonicimaging. sagepub.com.

Table 2. The Mean Maximum Displacement and Standard Deviation Values for $n=10$ Trials. Elastic Moduli of the Three Tissue-mimicking Phantoms are Reported as Well.

\begin{tabular}{lccc}
\hline & Phantom A $(2.6 \mathrm{kPa})$ & Phantom B $(2.8 \mathrm{kPa})$ & Phantom C $(2.9 \mathrm{kPa})$ \\
\hline $\begin{array}{l}\text { Mean maximum } \\
\text { displacement }(\mu \mathrm{m})\end{array}$ & 0.65 & 0.57 & 0.55 \\
$\mathrm{SD}$ & 0.22 & 0.13 & 0.11 \\
\hline
\end{tabular}

phased to produce a focused radiation force beam on a desired target close to the vessel endothelium. Physically short-circuiting a portion of the transducer circular array allowed us to demonstrate feasibility by generating acoustic radiation forces and tracking the resulting displacements from a diverging wave. Power spectrum analysis indicated spectral peaks at $8 \mathrm{MHz}$ and 20.5 MHz for the two IVUS prototypes. Hydrophone measurements for the Volcano Visions prototype and Volcano Eagle Eye Platinum prototype verified the potential for the generation of acoustic radiation forces with $I_{\mathrm{SPPA}}$ values similar to the positive control ATL L12-5 linear array transducer.

Results from the ARFI experiments are promising for the implementation of IVUS ARFI in carotid and coronary arteries. The modified Volcano Visions prototype demonstrated greater displacement than the L12-5 transducer with a similar transmit aperture. Although the Volcano Eagle Eye Platinum prototypes tracked ARFI displacements, the displacement profiles lacked the smooth 
and well-defined displacements typically observed with ARFI imaging due to poor SNR and significant transducer ring down to an axial depth of $1 \mathrm{~mm}$. However, a comparison of Figure 8 to the control displacement profiles in Figures 6 and 7 shows that the $20 \mathrm{MHz}$ prototype provided enough acoustic radiation force to generate a typical ARFI temporal response. However, the ARFI measurements had adequate correlations over a depth window of only a few millimeters. Further, the phantoms used for these experiments had stiffness values similar to the lipid pools found in vulnerable plaques. Future experiments should involve implementation of ARFI imaging on both softer and harder phantoms with Young's Moduli ranging from 1 to $5 \mathrm{kPa}$ - the characteristic stiffness range of soft tissues in vulnerable plaques. ${ }^{34}$ Our attempts to produce softer phantoms failed due to the poor reproducibility in current fabrication methods.

Because of the noisy displacement tracking with the transmitted diverging wave, adjustments to the Volcano imaging system should be considered. We note that the 1 micron motion from the Volcano Eagle Eye was approaching the Cramér-Rao lower bound for detectable displacements for ARFI. ${ }^{35}$ Software code modifications of the Volcano scanner would allow electronic phasing of a portion of the elements for focused ARFI imaging at a desired location on the vessel endothelium. This would improve SNR, reduce electronic ring down, and provide a much stronger acoustic radiation force, resulting in improved detection and estimation of displacements and would permit imaging the mechanical properties of a wider range of tissues. Other possibilities would include ARFI synthetic aperture approaches.

The higher power levels required for ARFI could be achieved through hardware modifications to the Volcano scanner power supply and alterations to the integrated circuits of the commercially available imaging catheters, although it is uncertain whether transmit voltages approaching 200 Volts can be achieved. Our studies demonstrated that the catheter transducers can handle such high-voltage levels and extended pulse durations over multiple experiments and about 50 trials before failure. Analysis of the specturm of the catheter transducers has shown that its spectrum is unchanged between trials.

The flow of blood in the artery will not impact the radiation force generated by the catheter transducers. In fact, blood flow would improve IVUS ARFI in the sense that flowing blood would decrease both heating of the transducer and localized tissue heating near the transducer through convective heat transfer. Attenuation due to scattering from the blood itself may impede the generation of radiation force by the higher frequency catheter transducers, although the distances propagated in this domain are very small, so will limit the impact of attenuation compared with noninvasive applications of ARFI elsewhere in the body.

Coronary atherosclerotic disease is a major cause of fatal cardiovascular disease in the United States. Clinical decision-making and interventional treatment strategies for the disease rely on a sufficient understanding of atherosclerotic plaque composition in the coronary arteries. Vulnerable plaques are more likely to induce acute myocardial infarction and have a characteristic soft core surrounded by a tough, fibrous cap. In this study, we successfully demonstrated the potential for an acoustic radiation force from an endovascular approach to distinguish plaque morphology using circular array IVUS imaging catheters.

\section{Declaration of Conflicting Interests}

The author(s) declared no potential conflicts of interest with respect to the research, authorship, and/or publication of this article.

\section{Funding}

The author(s) received the following financial support for the research, authorship, and/or publication of this article: Funding was through the HL089507 and R01-EB013361. The author(s) also acknowledge in kind support from Volcano Corp. 


\section{References}

1. Perk G, Lang RM, Garcia-Fernandez MA, Lodato J, Sugeng L, Lopez J, et al. Use of real time threedimensional transesophageal echocardiography in intracardiac catheter based interventions. J Am Soc Echocardiogr. 2009;22:865-82.

2. Finn AV, Nakano M, Narula J, Kolodgie FD, Virmani R. Concept of vulnerable/unstable plaque. Arterioscl Throm Vas. 2010;30:1282-92.

3. Madjid M, Zarrabi A, Litovsky S, Willerson JT, Casscells W. Finding vulnerable atherosclerotic plaques - is it worth the effort? Arterioscl Throm Vas. 2004;24:1775-82.

4. Zunker P, Wilms H, Brossmann J, Georgiadis D, Weber S, Deuschl G. Echo contrast-enhanced transcranial ultrasound - frequency of use, diagnostic benefit, and validity of results compared with MRA. Stroke. 2002;33:2600-3.

5. Fayad ZA, Fuster V. Clinical imaging of the high-risk or vulnerable atherosclerotic plaque. Circ Res. 2001;89:305-16.

6. Tearney GJ, Yabushita H, Houser SL, Aretz HT, Jang IK, Schlendorf KH, et al. Quantification of macrophage content in atherosclerotic plaques by optical coherence tomography. Circulation. 2003;107:113-9.

7. Ge J, Chirillo F, Schwedtmann J, Gorge G, Haude M, Baumgart D, et al. Screening of ruptured plaques in patients with coronary artery disease by intravascular ultrasound. Heart. 1999;81:621-7.

8. Lovett JK, Redgrave JNE, Rothwell PM. A critical appraisal of the performance, reporting, and interpretation of studies comparing carotid plaque imaging with histology. Stroke. 2005;36:1085-91.

9. Touze E, Toussaint JF, Coste J, Schmitt E, Bonneville F, Vandermarcq P, et al. Reproducibility of high-resolution MRI for the identification and the quantification of carotid atherosclerotic plaque components consequences for prognosis studies and therapeutic trials. Stroke. 2007;38:1812-9.

10. Sabetai MM, Tegos TJ, Nicolaides AN, Dhanjil S, Pare GJ, Stevens JM. Reproducibility of computerquantified carotid plaque echogenicity — can we overcome the subjectivity? Stroke. 2000;31:2189-96.

11. Back MR, Bowser AN, Johnson BL, Schmacht D, Zwiebel B, Bandyk DF. Patency of infrarenal aortic side branches determines early aneurysm sac behavior after endovascular repair. Ann Vasc Surg. 2003; 17:27-34.

12. Nishioka T, Nagai T, Luo H, Kitamura K, Hakamata N, Akanuma M, et al. Coronary remodeling of proximal and distal stenotic atherosclerotic plaques within the same artery by intravascular ultrasound study. Am J Cardiol. 2001;87:387-91.

13. Nissen SE, Yock P. Intravascular ultrasound — novel pathophysiological insights and current clinical applications. Circulation. 2001;103:604-16.

14. Fitzgerald PJ, Yock PG. Mechanisms and outcomes of angioplasty and atherectomy assessed by intravascular ultrasound imaging. J Clin Ultrasound. 1993;21:579-88.

15. Wang B, Karpiouk A, Emelianov S. Design of catheter for combined intravascular photoacoustic and ultrasound imaging. Ultrasonics. 2008;1150-3.

16. Karpiouk AB, Wang B, Emelianov SY. Development of a catheter for combined intravascular ultrasound and photoacoustic imaging. Rev Sci Instrum. 2010;81:014901-1 to 014901-7.

17. Nightingale KR, Palmeri ML, Nightingale RW, Trahey GE. On the feasibility of remote palpation using acoustic radiation force. J Acoust Soc Am. 2001;110:625-34.

18. Eyerly SA, Bahnson TD, Koontz JI, Bradway DP, Dumont DM, Trahey GE, et al. Intracardiac acoustic radiation force impulse imaging: a novel imaging method for intraprocedural evaluation of radiofrequency ablation lesions. Heart Rhythm. 2012;9:1855-62.

19. Hsu SJ, Hubert JL, Smith SW, Trahey GE. Intracardiac echocardiography and acoustic radiation force impulse imaging of a dynamic ex vivo ovine heart model. Ultrason Imaging. 2008;30:63-77.

20. Hollender PJ, Wolf PD, Goswami R, Trahey GE. Intracardiac echocardiography measurement of dynamic myocardial stiffness with shear wave velocimetry. Ultrasound Med Biol. 2012;38:1271-83.

21. Doherty JR, Dumont DM, Hyun D, Dahl JJ, Trahey GE.Development and evaluation of pulse sequences for freehand ARFI imaging. In: Proceedings of the 2011 IEEE International Ultrasonics Symposium (IUS); 2011 Oct 7-10; Dresden, Germany. Piscataway. IEEE. 2013;P. 1281-4.

22. Dahl JJ, Dumont DM, Allen JD, Miller EM, Trahey GE. Acoustic radiation force impulse imaging for noninvasive characterization of carotid artery atherosclerotic plaques: a feasibility study. Ultrasound Med Biol. 2009;35:707-16. 
23. Turnbull DH, Foster FS. Beam steering with pulsed 2-dimensional transducer Arrays. IEEE Trans Ultrason Ferroelectr Freq Control. 1991;38:320-33.

24. Patel V, Light E, Herickhoff C, Palmeri M, Grant G, Britz G, et al. Intracranial dual-mode IVUS and hyperthermia using circular arrays preliminary experiments. In: Proceedings of the 2011 IEEE International Ultrasonics Symposium (IUS), October 7-10, 2011; Dresden, Germany. Piscataway: IEEE; 2013; P. 2185-8.

25. Light E, Smith SW. Two dimensional arrays for real time 3D intravascular ultrasound. Ultrason Imaging. 2004;26:115-28.

26. Lee W, Idriss SF, Wolf PD, Smith SW. A miniaturized catheter 2D array for real-time, 3D intracardiac echo cardiography. IEEE Trans Ultrason Ferroelectr Freq Control. 2004;51:1334-46.

27. Lee W, Idriss SF, Wolf PD, Smith SW. Dual lumen transducer probes for real-time 3D interventional cardiac ultrasound. Ultrasound Med Biol. 2003;29:1297-304.

28. Light ED, Angle JF, Smith SW. Real-time 3-D ultrasound guidance of interventional devices. IEEE Trans Ultrason Ferroelectr Freq Control. 2008;55:2066-78.

29. Patel V, Light E, Herickhoff CD, Grant GA, Britz G, Wilson C, et al. Intracranial dual-mode IVUS and hyperthermia using circular arrays: preliminary experiments. Ultrason Imaging. 2013;35:17-29.

30. Cobbold RSC. Foundations of Biomedical Ultrasound. New York: Oxford University Press; 2007.

31. Rouze NC, Wang MH, Palmeri ML, Nightingale KR. Robust estimation of time-of-flight shear wave speed using a radon sum transformation. IEEE Trans Ultrason Ferroelectr Freq Control. 2010;57:2662-70.

32. Palmeri ML, Wang MH, Dahl JJ, Frinkley KD, Nightingale KR. Quantifying hepatic shear modulus in vivo using acoustic radiation force. Ultrasound Med Biol. 2008;34:546-58.

33. Pinton GF, Dahl JJ, Trahey GE. Rapid tracking of small displacements with ultrasound. IEEE Trans Ultrason Ferroelectr Freq Control. 2006;53:1103-17.

34. Le Floc'h S, Ohayon J, Tracqui P, Finet G, Gharib AM, Maurice RL, et al. Vulnerable atherosclerotic plaque elasticity reconstruction based on a segmentation-driven optimization procedure using strain measurements: theoretical framework. IEEE Trans Med Imaging. 2009;28:1126-37.

35. Walker WF, Trahey GE. A fundamental limit on delay estimation using partially correlated speckle signals. IEEE Trans Ultrason Ferroelectr Freq Control. 1995;42:301-8. 